\title{
Aspectos clínico-epidemiológicos associados ao câncer de pênis
}

\author{
Clinico-epidemiological aspects associated with penile cancer
}

Angela Adamski da Silva Reis ${ }^{1}$

Leonardo Barcelos dePaula ${ }^{2}$

Adriano Augusto Peclat de Paula ${ }^{3}$

VeraA parecida Saddi ${ }^{4}$

Aparecido Divino da $\mathrm{Cruz}^{5}$

\footnotetext{
${ }^{1}$ Departamento deBiologia, Pontifícia Universidade Católica de Goiás. 1ậ Av. 1440, Área IV, Bloco L, Setor Universitário. 74605010 Goiânia GO . angeladamski@gmail.com ${ }^{2}$ Centro Regional de Hemoterapia, Hemocentro deRibeirão Preto,

Universidade de São Paulo.

${ }^{3}$ Serviço de Urologia do

H ospital Araújo J orge da

Associação de Combateao

Câncer em Goiás (ACCG).

${ }^{4}$ Pró-Reitoria de Pós-

Graduação ePesquisa,

Pontifícia Universidade

Católica de Goiás.

${ }^{5}$ Núcleo de Pesquisas

Replicon, Departamento de

Biologia, Pontifícia

Universidade Católica de

Goiás.
}

Abstract The general objective of this article is to review the current literature regarding the epidemiology, biological behavior and risk factors for penile cancer development, such as H PV infection. Phimosis and chronic irritation related to poor hygiene are commonly associated with penilecancer, whereas neonatal circumcision reduces the relative risk for the disease. There is strong evidence that H PV types 16 and 18 are associated with penilecarcinoma in as many as $50 \%$ of cases. Patients with penile lesions should undergo physical examination, which is often sufficient to diagnose and to define tumor stagging, as well as contributes to decision-making regarding therapeutical approaches and case management.

Key words Penile cancer, Risk factor, Human papillomavirus (HPV), Epidemiology
Resumo 0 objetivo desta revisão bibliográfica foi descrever a epidemiologia, o comportamento biológi co dos tumores e os fatores de risco para o câncer de pênis, como a infecção pelo H PV. A fimosee os processos de irritação crônica relacionados à má higiene estão comumente associados com esse tumor, enquanto a circuncisão neonatal protege contra a aparição da doença. Existe forte evidência da associação dos H PV 16 e 18 com o carcinoma peniano em mais de $50 \%$ dos casos. Pacientes com lesões penianas suspeitas devem se submeter ao exame físico, geralmente sendo este suficiente para determinar o diagnóstico e o estadiamento, assim como auxiliar na escolha terapêutica.

Palavras-chave Câncer de pênis, Fatores derisco, Papilomavírus humano (HPV), Epidemiologia 
Introdução

0 câncer depêniséuma neoplasia rara, queatinge aproximadamente $1 / 100.000$ homens nos países desenvolvidos ${ }^{1,2}$. A alta incidência é observada em países em desenvolvimento, incluindo 0 Brasil, onde é mais elevada nas regiões $\mathrm{N}$ orte e Nordeste ${ }^{3}$, acometendo principalmente homens na terceira idade, independentemente de sua origem étnica ${ }^{1,4}$. No entanto, indivíduos jovenstambém podem ser afetados, uma vez que aproximadamente $22 \%$ dos casos são registrados em pacientes com idades inferiores a quarenta anos. A doença acometeindivíduos de baixo nível social, com maus hábitos de higiene e não circuncizados, tendo como principal fator de risco a fimose, e muitas vezes está associada ao papilomavírus humano (HPV) ${ }^{4}$.

A infecção pelo HPV éa doença sexualmente transmissível encontrada com mais frequência entre os indivíduos sexualmente ativos, envolvendo diversos fatores de risco ${ }^{5}$. Estudos epide miológicos têm demonstrado a associação etiológica entre o H PV e o carcinoma de cérvice uterina ${ }^{6-8}$, sendo o homem considerado um importante fator propagador do vírus ${ }^{9}$.

0 avanço das técnicas de detecção moleculares possibilita a detecção do genoma do H PV em diversos tecidos, incluindo as células neoplásicas malignas penianas ${ }^{10,11}$. A presença do HPV em carcinomas de pênis foi demonstrada pela primeira vez, no Brasil, na década de oitenta, por Villae Lopes ${ }^{12}$. Estudos moleculares demonstram que mais de $50 \%$ dos carcinomas de pênis apresentam DNA de HPV, prevalecendo os tipos virais oncogênicos 16 e 18 $8^{13,14}$. Contudo, a carência em estudos sobre a associação entre H PV e carcinomas penianos é grande, devido à neoplasia ser considerada rara.

\section{Epidemiologia efatores de risco}

Em países onde a circuncisão neonatal éum hábito cultural, verifica-se que a incidência do carcinoma de células escamosas do pênis (CCE) é baixa ${ }^{2,15,16}$. A higiene adequada e a circuncisão precoce previnem a ocorrência da neoplasia na idade adulta. A história de fimose é encontrada em aproximadamente $85 \%$ dos pacientes com câncer de pênis, estando associada às lesões pré cancerígenas ${ }^{2,4}$.

Os tipos histológicos mais comuns de câncer de pênis incluem o carcinoma de origem escamosa eo carcinoma in situ. Esteúltimo éconhe cido como eritroplasia de Queyrat e doença de Bowen, que se apresentam na forma de modificações celulares epiteliais não invasivas²,17. 0 CCE é o tipo histológico mais comum e representa $95 \%$ dos casos, sendo que seu prognóstico relaciona-se com o estadiamento do tumor ${ }^{1,2,4,17}$.

Estudos em câncer de pênis têm demonstrado a associação do HPV com lesões benignas e malignas ${ }^{3,4}$. A associação entre infecção por H PV e tumor peniano levanta considerações sobre 0 papel do HPV na etiologia do câncer de pênis. Evidências sugerem quepacientesinfectadoscom os tipos virais oncogênicos 16, 18, 31 e 33 apresentam uma predisposição para o desenvolvimento do CCE ${ }^{3,17-19}$. Em muitos indivíduos, o HPV causa o condiloma acuminado. Entretanto, alguns homens podem ser portadores totalmente assintomáticos do vírus, enquanto outros abrigam lesões intrauretrais desconhecidas ao próprio paciente, tornando-se uma fonte potencial de transmissão para parceiros sexuai ${ }^{14}$.

O HPV é capaz de alterar o ciclo celular pela expressão das proteínas virais E6 eE7 na inativação e na eliminação dos produtos de genes supressores de tumor ( $p 53$ e Rb). A perda das funções de ambas as proteínas responsáveis pela supressão tumoral contribui para a progressão do processo neoplásico. Em geral, a atividade de $\mathrm{Rb}$ é inibida pela proteína viral $\mathrm{E} 7$, enquanto a p53 é degradada subsequentementeà ligação com a proteína viral E6 ${ }^{7,20,21}$. As interações entre as proteínas virais eas proteínas celulares al teram 0 controle do ciclo celular, fazendo da infecção viral um potente fator de iniciação e promoção de tumores. Todas as variáveis envolvidas na carcinogênese devem ser avaliadas em conjunto, a fim de estabelecer os riscos relativos e o prognóstico da infecção?.

O Brasil é considerado um país de alta incidência decâncer de pênis, sendo os tipos virais 16 e18 os mais prevalentes ${ }^{22,23}$. Villa e Lopes ${ }^{12}$ detectaram a presença de H PV em $44 \%$ das amostras de carcinomas penianos, avaliadas por Southern blotting, sendo os HPVs 18 e 11 os tipos virais encontrados. Dados da literatura apoiam a associação do genoma do H PV com os casos de câncer de pênis, demonstrando a presença do vírus em 15 a 71\% dos casos ${ }^{2,14,22-25}$. No entanto, em função da existência dos diversos subtipos virais, sugere-se que outros tipos de HPVs devem ser avaliados para o esclarecimento da patogênese nos tumores de pênis.

Adicionalmente, é necessário intensificar as campanhas de preven ção, repassando à população em geral o conhecimento sobre a associação 
dos maus hábitos dehigiene eo efeito carcinogê nico da fimose e da infecção pelo H PV. Campanhas de preven ção podem diagnosticar o câncer depênis nos estágiosiniciais, reduzir a incidência e a severidade da doença, como também proporcionar maiores chances de cura e aumento da sobrevida ${ }^{10}$.

\section{Estadiamento do câncer de pênis}

O sistema de classificação eestadiamento do câncer foram propostos devido à necessidade de se uniformizar a terminologia utilizada. A correta descrição clínica e a classificação histopatológica das neoplasias malignas auxiliam os profissionais no planejamento do tratamento, oferecem indicação do prognóstico, auxiliam na avaliação dos resultados do tratamento e, ainda, contribuem para a pesquisa contínua acerca da doença $^{26}$. 0 sistema de estadiamento TNM oferece informações relacionadas ao tamanho do tumor, à quantidadeetamanho dos nódulos regionais acometidos e, finalmente, à presença de metástase à distância. As informações combinadas sobre tumor, nódulos linfáticos e metástase determinam 0 estadiamento, que é descrito em números romanos, variando de I a IV ${ }^{27,28}$. Dois sistemas são usados em câncer depênis, incluindo a classificação de Jackson ${ }^{18}$ (Tabela 1) e o sistema TNM ${ }^{29}$ (Tabela 2).

Tabela 1. Classificação de Jackson para o estadiamento do câncer de pênis.

\begin{tabular}{ll}
\hline Estágio I & $\begin{array}{l}\text { Encontra-se circunscrito à glande e ao prepúcio, sem envolvimento do corpo do pênis ou do } \\
\text { corpo cavernoso. } \\
\text { Apresenta invasão do corpo cavernoso do pênis, mas sem disseminação para os linfonodos, } \\
\text { Estágio II }\end{array}$ \\
Estágio III & $\begin{array}{l}\text { Apresenta disseminação clínica nos linfonodos regionais da virilha. A possibilidade de cura } \\
\text { depende do número e da extensão dos nodos envolvidos. }\end{array}$ \\
Estágio IV & $\begin{array}{l}\text { É de natureza invasiva, apresentando extenso envolvimento dos linfonodos, sem } \\
\text { possibilidade de intervenção cirúrgica, na virilha e/ou metástases distantes. }\end{array}$
\end{tabular}

Tabela 2. Sistema TNM - proposto pela UICC/AJCC para estadiamento do câncer de pênis.

\begin{tabular}{ll}
\hline \multicolumn{1}{c}{ Estágio } & \multicolumn{1}{c}{ D escrição } \\
\hline Tumor (T) & Tumor primário não é passível de avaliação \\
Tx & Sem evidência de tumor primário \\
T0 & Carcinoma in situ \\
Tis & Tumor verrucoso não invasivo \\
Ta & Tumor invade tecido conectivo subepitelial \\
T 1 & Tumor invade corpo cavernoso \\
T2 & Tumor invade a uretra ou a próstata \\
T3 & Tumor invade outras estruturas adjacentes \\
T 4 & \\
Linfonodos regionais (N) & Linfonodos regionais não são passíveis de avaliação \\
N X & Sem evidências de infiltração \\
N 0 & M etástase unical \\
N 1 & M etástase múltiplas ou bilaterais superficiais \\
N 2 & M etástase em linfonodos profundos pélvicos ou inguinais \\
N 3 & \\
M etástase distante (M) & Presença de metástases distantes não é passível de avaliação \\
M X & Nenhuma metástase distante \\
M 0 & M etástase à distância \\
M 1 &
\end{tabular}




\section{Apresentação clínica e tratamento}

A queixa do paciente com câncer de pênis é comumente relacionada à presença de lesão vegetante ou deáreas de ulceração peniana. As lesões variam quanto às dimensões, e, com frequência, o paciente procura 0 atendimento médico tardiamente, por falta de recursos locais ou mesmo por temer o tratamento cirúrgico ${ }^{30}$.

Como o pênis é formado por tecidos que incluem pele, nervos, musculatura e vasos sanguíneos, a apresentação clínica do tumor depende das diferenças histológicas presentes no órgão. Assim, diferentes tipos de neoplasias podem ser detectados de acordo com a célula afetada. A diferença celular em nível histológico determina 0 tipo de câncer, como também o tratamento ${ }^{4}$. A primeira etapa no diagnóstico histológico da malignidade é a confirmação do diagnóstico e a avaliação da profundidade da invasão por biópsia e análise de microscopia ${ }^{4,31}$.

A fimose pode obscurecer o tumor, mascarando o diagnóstico. Em geral, a sintomatologia inclui o sangramento e mau cheiro da genitália. Todas as lesões penianas, particularmente aquelas em que o paciente apresenta o prepúcio não retrátil, requerem uma atenção especial e devem ser investigadas com suspeita de neoplasia. A biópsia nesses casos é uma importante forma de diagnóstico. Em lesões grandes, o diagnóstico é obtido por biópsia incisional4,32.

0 tratamento do câncer de pênis é baseado na extensão do tumor primário e na sua classificação, estabelecidas pela análise histopatológica a partir da biópsia da lesão. A terapia antibiótica inicia-se antes da biópsia e continua com a terapia cirúrgica, estendendo-se por quatro a seis semanas. Uma vez que o diagnóstico do tecido é confirmado, os tumores superficiais pequenos podem, com sucesso, ser tratados por meio de excisão ci rúrgica local, quimioterapia, cirurgia a laser ou terapia de radiação superficial 33,34 .

As lesões metastáticas que envolvem o corpo cavernoso são ocasionadas frequentemente por nódulos múltiplos e pal pávei ${ }^{30,35-37}$. 0 tratamento de tumores com extensão maior e invasão dos nódulos regionais é controlado com cirurgia ou radioterapia. Porém, tumores profundamente invasivos, particularmenteaqueles com lesõesque envolvem o eixo distal ou que deformam a glande, são controlados geralmente por penectomia parcial. Lesões mais extensivas ou tumores que envolvam a base ea parte uretral bulbar do pênis são controlados por penectomia total ${ }^{30}$ ou requerem cistoprostatectomia, ou, ocasional mente, es- vaziamento pélvico anterior ou total, com diversificação urinária. Os pacientes que apresentam metástase inguinal devem ser submetidos à linfadenectomia pélvica na região afetada ${ }^{36,37}$.

É fato conhecido queaproximadamente $20 \%$ dos tumores de pênis apresentam metástases linfonodais. Em contrapartida, 50\% dos linfonodosclinicamentecomprometidosnão têm tumor e sim reação inflamatória ${ }^{30}$. É recomendável que sejam feitos cortes de congelação durante as linfadenectomias, o que poderia implicar o aumento da extensão da dissecção ${ }^{38}$. As linfadenectomias inguinais parciais (linfonodos sentinelas ou superficiais), quando realizadas, são acompanhadas de estudo histopatológico, com o objetivo de avaliar o estadiamento e sugerir o tratamento terapêutico adequado ${ }^{39}$. O s cortes de congelação devem informar sobre a eventual presença de células neoplásicas, eo laudo definitivo do material deve ser elaborado posteriormente ${ }^{30,38}$.

Os tumores penianos tendem a evoluir de maneiralenta, inicialmentesuperficial, invadindo a seguir o córion, o tecido esponjoso da glande e os corpos cavernosos. A infiltração da uretra é rara e, em geral, ocorre apenas nas fases avançadas da evolução dos tumores dos corpos cavernosos. 0 comportamento biológico desses tumores tende a ser uniforme, com disseminação para linfonodosinguinais, linfonodos pélvicose periaórticos, e raramente apresentam comprometimento visceral. Os pacientes não tratados ou cujo tratamento foi ineficaz, em geral, morrem por complicações secundáriasàs metástases inguinais, ou seja, hemorragias por lesões tumorais degrandes vasos ou processos sépticos ${ }^{36,38}$.

A disseminação tumoral tem relação com as características histopatológicas da lesão primária. É fato reconhecido que lesões de grandes dimensões e com histologia desfavorável têm mai or tendência à disseminação. 0 conhecimento desses aspectos histopatológicos tem importância fundamental no planejamento terapêutico, assim como no prognóstico dos pacientes. A padronização do exame anatomopatológico, assim como sua interpretação, éindispensável para o serviço clínico ${ }^{30}$.

Estudos recentes confirmam a importância da classificação do tumor edo estadiamento para auxiliar no prognóstico e na terapêutica. Os tumores de classe I, limitados à pele e aos tecidos superficiais do pênis, são improváveis de se espalhar para a região inguinal. No entanto, as lesões de classe II ou III, com qualquer grau de invasão na estrutura peniana, possuem um risco significativo de metástases em linfonodos ingui- 
nais eilíacos, caracterizando a disseminação ge neralizada ${ }^{17,39}$.

O diagnóstico, na maioria dos casos, é realizado em estágios avançados, sendo observada uma sobrevida geral baixa. Essetipo particular de câncer apresenta portadores que vivenciam um processo defragilização psicológica típica, devido ao binômio câncer de pênis e mutilação. 0 abandono do tratamento é um evento comum nesse tipo de câncer, sobretudo pelo fato de o tratamento de escolha ser a penectomia. 0 paciente penectomizado, no âmbito psicológico, perde a sua referência de masculinidade e, comumente, não retorna ao serviço de saúde para dar seguimento ao tratamento e controle da doença33.

\section{Conclusão}

Considera-se fator de risco, para uma determinada doença, todo e qualquer fator que contribui para o surgimento dessa enfermidade. No entanto, a ocorrência de um ou mais fatores de risco não implica, necessariamente, na presença da doença. Para o câncer de pênis, epidemiologicamente, a não-realização de circuncisão, a presença de fimose, a higiene inadequada, as infecções virais e o comportamento sexual de risco são os principais fatores para o desenvolvimento da neoplasia $a^{4}$. 0 fato de a incidência de câncer de pênis ser maior em países em desenvolvimento do queem países desenvolvidos deve-se às cir- cunstâncias socioeconômicas, além da dificuldade de acesso à saúde.

De todos os tumores urológicos, o carcinoma peniano constitui a analogia mais próxima do câncer de colo uterino. Estudos epidemiológicos demonstram estreita correlação entre os dois tipos de câncer. Mulheres cujos parceiros apresentam câncer de pênis possuem risco 2,8 a 3,2 vezes mais elevado para o desenvolvimento do câncer de colo uterino ${ }^{40-42}$. Tais achados revelam o HPV como agente etiológico do carcinoma de cérvice uterina, tornando provável a associação entre o H PV e o câncer de pênis.

A melhor compreensão da histologia do tumor confere uma avaliação mais precisa para o prognóstico e a terapêutica da doença. 0 tratamento da neoplasia ésemprecirúrgico, com retirada da lesão e, em alguns casos, com a amputação parcial do membro e a retirada dos gânglios da região inguinal (primeiro sítio de metástase dessa doença). 0 diagnóstico precoce é fundamental para evitar o desenvolvimento da doença e a amputação, que acarretam consequências físicas, sexuais e psicológicas para o paciente.

0 progresso no entendimento da etiologia e patogênese e o prognóstico mais acurado dos tumores malignos de pênis têm sido lentos devido à carência deestudos moleculares que investiguem as prováveis alterações genéticas, agentes biológicos e clínicos associados ao desenvolvimento e progressão dessa neoplasia.

\section{Colaboradores}

AAS Reis, LB Paula, AAP Paula, VA Saddi e AD Cruz participaram igualmente de todas as etapas da elaboração do artigo. 
1. Velazquez EF, Cubilla AL. Penile squamous cell carcinoma: anatomic, pathologic and viral studies in Paraguay (1993-2007). Anal Quant Cytol Histol 2007; 29(4):185-198.

2. Micali G, Nasca MR, Innocenzi $D$, Schwartz RA. Penile Cancer. J Am Acad Dermatol 2006; 54:369-391.

3. Gil AO, Pompeo AC L, Golstein PJ, Saldanha LB, M esquita JLB, Arap S. Analysis of the association between Human Papillomavirus with penile carcinoma. Braz J U rol 2001; 27(5):461-468.

4. Pow-Sang M R, Benavente V, Pow-Sang JE, M orante $C$, M eza L Baker M, Pow-Sang JM. Cancer of penis. Canc Contr J 2002; 9(4):305-314.

5. Teixeira JC, Derchain SFM, Teixeira LC, Santos CC, Panetta K, Zeferino LC. Avaliação do parceiro sexual e risco de recidivas em mulheres tratadas por lesões genitais induzidas por Papilomavírus $\mathrm{Hu}$ mano (HPV). Rev Bras Ginecol Obstetrícia 2002; 24(5):315-320.

6. Yu T, Ferber MJ, Cheung TH, Chung TK, Wong YF, Smith DI. The role of viral integration in the development of cervical cancer. Canc Genet Cytogenet 2005; 158(1):27-34.

7. Boccardo E, Villa LL. Vírus e Câncer. In: Ferreira CG, Rocha JCC. Oncologia molecular. São Paulo: Atheneu; 2004. p. 123-132.

8. Burd EM . Human Papillomavirus and Cervical Cancer. Am Society for Microbiol 2003; 16:1-17.

9. Baldwin SB, Wallace DR, Papenfuss MR, Abrahansen $M$, Vaught LC, Kornegay JR, Hallum JA, Redmond SA, Giuliano AR. Human papillomavirus infections in men attending a sexually transmittted disease clinic. J Infect Dis 2003; 187:1064-1070.

10. Hart KW, Williams OM, Thelwell N, Fiander AN, Brown T, Borysiewicz LK, Gelder CM. Novel method for detection, typing and quantification of human papillomavirus in clinical samples. J Clin Microbiol 2001; 39(9):3204-3212.

11. Elnifro E, Asshi AM, Cooper RJ, Klapper PE. Multiplex PCR: Optimization and application in diagnostic virology. Clin Microbiol Rev 2000; 13(4):559-570.

12. Villa LL, Lopes A. Human Papillomavirus DNA sequence in penile carcinomas in Brazil. Int J Canc 1986; 37:853-855.

13. Chan KW, Lam KY, Chan ACL, Lau P, Srivastava G. Prevalence of human papillomavirus types 16 and 18 in penile carcinoma: a study of 41 cases using PCR. J Clin Pathol 1994; 47:823-826.

14. McCance DJ, Kalache A, Ashdown K, Andrade L, Menezes F, Smith P, Doll R. Human papillomavirus types 16 and 18 in carcinomas of the penis from Brazil. Int J Can 1986; 37(1):55-59.

15. Castellsagué $X$, Bosch FX, M uñoz N, M eijer CJLM, Shah KV, Sanjosé A, Eluf- $N$ eto J, N gelangel CA, Chichareon S, Smith JS, Herrero R, Moreno V, Franceschi S, International Agency for Research on Cancer Multicenter Cervical Cancer Study Group. $M$ ale circumcision, penile human papillomavirus infection and cervical cancer in female partners. N Engl J M ed 2002; 346:1105-1112.
16. Schoen EJ, Oehrli M, Colby CJ, Machin G. The highly protective effect of newborn circumcision against invasive penile cancer. Pediatr 2000; 105(3):36-40.

17. Ornellas $A A$, Brown GA. Câncer urológico (câncer de pênis). In: Ferreira CG, Rocha JC. Oncologia molecular. São Paulo: Atheneu; 2004. p. 224-229.

18. Cruz MR, Cerqueira DM, Cruz WB, Câmara GNL, Brígido M M, Silva EO, Carvalho LG, Martins CR. Prevalence of human papillomavirus type 16 variants in the Federal District, Central Brazil. Mem Inst O swaldo Cruz 2004; 99(3):281-282.

19. Tornesello ML, Buonaguro FM, Buonaguro L, Salatiello I, Giraldo EB, Giraldo G. Identification and functional analysis of sequence rearrangements in the long control region of human papillomavirus type 16 Af- 1 variantes isolated from U gandan penile carcinomas. J Gen Viro 2000; 81:2969-2982.

20. Herrero R, Castellsagué $X$, Pawlita $M$, Lissowska J, Kee F, Balaram P, Rajkumar T, Sridhar H, Rose B, Pintos J, Fernández L, Idris $A$, Sánchez MJ, Nieto A, Talamini R, Tavani A, Bosch FX, Reidel U, Snijders PJ, Meijer CJ, Viscidi R, M uñoz N, Franceschi S, IARC Multicenter Oral Cancer Study Group. Human Papillomavirus and Oral Cancer: The International Agency for Research on Cancer Multicenter Study. J Natl Canc Inst 2003; 95(23):1772-1783.

21. Villa LL. Aspectos moleculares da oncogênese por papilomavírus. In: Bibbo M, Silva Filho AS. Lesões relacionadas à infecção por H PV no trato anogenital. Rio de Janeiro: Revinter; 1998. p. 51-58.

22. Neves D, Camara GNL, Alencar TR, da Cruz MR, Martins CRF, Carvalho LGS. Prevalence of human papillomavirus in penile carcinoma. Braz J U rol 2002; 28(3):221-226.

23. Gil AO, Pompeo AC L, Golstein PJ, Saldanha LB, $M$ esquita JLB, Arap S. Analysis of the association between Human Papillomavirus with penile carcinoma. Braz J U rol 2001; 27(5):461-468.

24. Kiyabu MT, Shibata D, Armheim N, Martin WJ, Fitzgibbons PL. Detection of human papillomavirus in formalin-fixed, invasive squamous carcinomas using polymerase chain reaction. Am J Surg Pathol 1989; 13:221-224.

25. Picconi MA, Eiján AM, Distéfano AL, Pueyo S, Alonio LV, Gorostidi S, Teyssié AR, Casabé A. Human papillomavirus (HPV) DNA in penile carcinoma in Argentina: Analysis of primary tumors and lymph nodes. J M ed Virol 2000; 61:65-69.

26. Black RJ, Bashir BA. World standard cancer patient populations: resource for comparative analysis of survival data. In: Cancer Survival in Deleloping Countries. IARC Scien Publ 1998; 145-9-11.

27. International Union Against Cancer. TNM Classification of Malignant Tumours. $6^{\text {th }}$ ed. New York: Wiley-Lis; 2002. p. 181-183.

28. Solsona E, Algaba F, H orenblas S, Pizzocaro G, Windahl T. Guidelines on penile cancer. European U rol 2004; 46(1):1-8.

29. Sobin LH, Wittekind $\mathrm{CH}$. TNM Classification of $M$ alignant Tumors. $6^{\text {th }}$ ed. New York: Wiley-Liss 2002. 
30. Pompeo ACL, Billis A. Carcinoma epidermóide do pênis. Uropatologia - Pênis. Int Braz J Urol 2003; 29:44-50.

31. Cubilla AL, Reuter VE, Gregoire L, Ayala GO, Campos S, Lancaster WD, Fair W. Basaloid squamous cell carcinoma: a distinctive human papillomavirus related penile neoplasm: a report of 20 cases. Am J Pathol 1998; 22:755-761.

32. Cubilla AL. Carcinoma of the penis. M od Pathol 1995; 8(1):116-118.

33. Holland JF, Frei E. Penile cancer. In: Kufe DW, Pollock RE, Weichselbaum RR, Bast Jr. RC, Gansler TS, Holland JF, Frei E, editors. Cancer M edicine 6. London: BC Decker Inc; 2003.

34. Blatstein LM, Finkelstein LH. Laser surgery for the treatment of squamous cell carcinoma of the penis. J Am Osteopathol Assoc 1990; 90(4):338-344.

35. Griffiths TRL, M ellon JK. Human Papillomavirus and U rological tumours: I. Basic science and role in penile cancer. Braz J U rol 1999; 84:579-586.

36. Srinivas V, Morse MJ, Herr HW, Sogani PC, Whitmore WF Jr. Penile cancer: relation of extent of nodal metastasis to survival. J U rol 1987; 137:880-882.

37. Lynch DF, Schellhammer PF. Tumors of the penis. In: Walsh PC, Retik AB, Vaughan ED, Wein AJ, Saunders WB, editors. Campbell's U rology. $7^{\text {th }}$ ed. Philadelphia: Saunders; 1998. p. 2453-2486.

38. McD ougal WS, Kirchner FK Jr, Edwards RH, Killion LT. Treatment of carcinoma of the penis: the case of primary lymphadenectomy. J U rol 1986; 136:38-41

39. Burgers JK, Badalament RA, Drago JR: Penile cancer. Clinical presentation, diagnosis, and staging. U rol Clin North Am 1992; 19:247-256.

40. Barasso R, De Brux J, Croissant O, Orth G. High prevalence of papillomavirus associaated penile intraephitelial neoplasia in sexual partners of women cervical intraephitelial neoplasia. N Engl J Med 1987; 317(15):916-923.

41. Castellsagué $X$, Ghaffari A, Bosch DFX, M uñoz N Shah KV for the International Agency for Research on Cancer (IARC) Multicentric Cervical Cancer Study Group. Prevalence of penile human papillomavirus DNA in Husband of women with and without cervical cancer neoplasia: a study in Spain and Colombia. J Inf Dis 1997; 176:353-361.

42. Graham S, Priore R, Graham M. Genital cancer in wives of penile cancer patients. Canc 1979; 44:18701874.

Artigo apresentado em 26/10/2007

Aprovado em 14/12/2007

Versão final apresentada em 15/01/2008 